\title{
Hemorrhagic uterine necrosis after surgical vessel ligation and B-Lynch suture in persistent post-cesarean uterine atony: case report and review of literature
}

\section{Giusti S. ${ }^{1}$, Fanti F. ${ }^{1}$, Cecchi E. ${ }^{2}$, Capriello P. $^{2}$, Malacarne E. $^{2}$, Marmorato ${ }^{2}{ }^{2}$, Vergine . $_{.}{ }^{\text {, }}$, Giannini A. ${ }^{1}$, Gadducci A. ${ }^{2}$, Caramella D. ${ }^{1}$, Simoncini T. ${ }^{2}{ }^{*}$}

${ }^{1}$ Division of Diagnostic and Interventional Radiology, University of Pisa, Italy
${ }^{2}$ Division of Obstetrics and Gynecology, University of Pisa, Italy

Received: 25 August 2016

Accepted: 24 September 2016

\section{*Correspondence:}

Dr. Simoncini T.

E-mail: tommaso.simoncini@med.unipi.it

Copyright: $(\odot$ the author(s), publisher and licensee Medip Academy. This is an open-access article distributed under the terms of the Creative Commons Attribution Non-Commercial License, which permits unrestricted non-commercial use, distribution, and reproduction in any medium, provided the original work is properly cited.

\section{ABSTRACT}

Uterine necrosis is a rare life-threatening condition reported in few case reports and series, associated with uterine artery embolization for uterine fibroids or postpartum hemorrhage. We report a hemorrhagic uterine necrosis in a nulliparous 35 years-old woman underwent cesarean section at 40+1 weeks of gestation for obstructed labor, presenting post-partum persisting bleeding and uterine atony and congestion. Bleeding stopped only after placement of two set of compressive sutures, curettage of uterine cavity and placement of Bakri Balloon but the uterine body never contracted and become congested. Notwithstanding an effective antibiotic therapy, the patient developed an intermittent fever and signs of severe anemia. Clinical and radiological diagnosis with CT and MRI scan were compatible with uterine necrosis characterized by hemorrhagic infarction of the uterine wall and decomposition of its muscular tissue. Hysterectomy was discussed with patient and performed on day 32 after C-section. Uterine apoplexy, a rare life-threatening disease, was detected with $\mathrm{CT}$ by lack of uterine contrast enhancement and a gas-filled uterine cavity and necrosis was confirmed with MRI by showing fluid degeneration of the myometrium. Failure to recognize a necrotic uterus on imaging can cause delayed hysterectomy, which is mandatory and potentially life-saving.

Keywords: Case report, CT, MRI, Hemorrhagic infarction, Uterine necrosis

\section{INTRODUCTION}

Uterine necrosis is a rare life-threatening condition reported only in few case reports and series. ${ }^{1}$ It can be associated with uterine artery embolization for uterine fibroids or postpartum hemorrhage and cesarean delivery. ${ }^{2-9}$ Regardless of etiology, prompt treatment with antibiotics and hysterectomy is mandatory to prevent septicemia. Because uterine necrosis is rare, there are few descriptions of the associated imaging findings.

We report the clinical, pathological CT and MRI findings of a woman incurred in hemorrhagic uterine necrosis after surgical vessel ligation and B-Lynch suture for postpartum hemorrhage due to untreatable uterine atony following cesarean section.

\section{CASE REPORT}

A nulliparous 35 years-old woman with gestational diabetes controlled with diet, was admitted to the obstetric ward of our hospital at $40+1$ weeks of gestation for premature rupture of the membranes. Her past medical history was uneventful except for an ectopic pregnancy with tubal abortion. Labor arose spontaneously; active labor went on for eight hours, then the woman underwent oxytocin augmentation due to ineffective contractions from $6 \mathrm{~cm}$ of dilatation and 
epidural analgesia from $7 \mathrm{~cm}$ of dilatation. After 2 hours cesarean section was performed because of obstructed labor, mostly due to persistent ineffective contractions notwithstanding oxytocin infusion. A healthy baby girl of 3490 g (5' Apgar: 9; Artery pH 7.27, BE 5.6) was born. The placenta was normally inserted on the posterior uterine wall and was easily detached.

Upon placental removal the uterus did not respond to the standard maneuvers to induce contraction, with a persistent and diffused bleeding. Oxytocin and sulprostone were administered, to no avail. A bilateral uterine artery ligation was performed, followed by placement of Hayman compressive sutures. However, complete atony and bleeding persisted, and the uterus became congested. The first set of compressive sutures was removed and after a curette revision a Bakri balloon (500 cc repletion) was positioned inside the uterine cavity after placement of a B-Lynch suture. Upon closure of the uterine incision and tightening of the B-Lynch suture the bleeding stopped. Total blood loss was around $800 \mathrm{cc}$. Notably, the uterine body never contracted. A pelvic drain was left in place before abdominal closure.

On the following day, hemoglobin level was $10.0 \mathrm{~g} / \mathrm{dl}$ and the vital signs were normal. The intrauterine as well as the pelvic drains were essentially dry. After an uneventful post-op day, on the second day post-surgery hemoglobin dropped rapidly to $4.5 \mathrm{~g} / \mathrm{dl}$ and the patient developed clinical signs of severe anemia. Both the uterine and pelvic drains kept being dry.

The patient was transfused with 3 UI red blood cells Units and a CT scan of the abdomen was performed. CT imaging showed an enlarged uterus that reached L2-L3 (190 mm craniocaudal diameter), containing the Bakri Baloon, with a hypodense low enhancing wall (thickness range $1.7-3.5 \mathrm{~cm}$ ) and presence of air, both in the uterine cavity and in the muscular tissue. There was no proof of ongoing active bleeding but the CT scan was suggestive of a massive intra-myometrial hemorrhagic infarction (Figure 2a).

The Bakri Balloon was removed 72 hours after the cesarean delivery. No significant vaginal bleeding was noted in the following days. Methylergometrine and oxytocin were administered, but the uterine size did not show any sign of physiological involution (the fundus reached $3 \mathrm{~cm}$ above the umbilical transverse line until day 32). Hemoglobin serum levels kept stable, with slight and slow decreases that required further four RBC transfusions during the following 30 days.

Notwithstanding an effective antibiotic therapy, the patient developed an intermittent fever, reaching max $38.5^{\circ} \mathrm{C}$ and lasting up to 32 days from the $\mathrm{C}$ section. CRP was always high $(\max 28.4 \mathrm{mg} / \mathrm{dl})$ with negative PCT and absent/low leukocytosis (max 16.470/mcl). Multiple blood cultures were negative, as were urine culture, PICC culture and tests for $\mathrm{CMV}$ and $\mathrm{C}$. difficile. Antibiotics were modified but with no response. Figure 1 depicts the body temperature changes during the hospital stay.

The patient felt well apart from the presence of fever and low-grade anemia. Pelvic examination revealed a persistently enlarged uterus and no pain during bimanual palpation. Hysterectomy was discussed, but in view of the woman's parity and young age a delay was attempted under close clinical observation. On day 21 another CT scan was performed, confirming persistent uterus enlargement (craniocaudal diameter was then $165 \mathrm{~mm}$ ) and an even lower density of its wall; intra-myometrial air was still present, and no active bleeding. Thickening of the surrounding adipose tissue and a thin layer of intraperitoneal effusion was also documented (Figure 2b). At trans-abdominal pelvic ultrasonography, the myometrium was irregular, with several hyperechoic spots and serpiginous anechoic images on the posterior wall that showed no flow at Power Doppler.

MRI was then performed to better understand the uterine condition. T2w sequences with and without fat saturation showed an irregularly hyperintense wall with some fluid content due to a completely infarcted myometrium (Figures 2c-3a).

Dixon T1w sequence was performed: in the fat images the uterus was completely hypointense (picture 5) and it was slightly hyperintense in the water images (Figures $3 b-3 c)$.

T1-weighted images (T1wi) confirmed the enlargement of the uterus, with thickened and irregular signal intensity of its wall; endometrial cavity was full of fluid with little gas.

Clinical and radiological diagnosis was compatible with uterine necrosis characterized by a complete hemorrhagic infarction of the uterine wall and complete decomposition of its muscular tissue, thus hysterectomy was performed on day 32 after C-section.

At surgical abdominal exploration as necrotic uterus was found, with multiple adherences to the bowel and anterior abdominal wall. Visual inspection and pathological exam after total hysterectomy confirmed the presence of a complete hemorrhagic necrosis of the uterus (Figure 4). The size of the uterus was $18 \times 15 \times 9.5 \mathrm{~cm}$ and the cervix was irregular. Microscopic examination confirmed extensive hemorrhagic necrosis of the entire uterus, associated with vascular congestion. No microorganism was cultured from the uterus.

Postoperative course was normal and the patient rapidly became (and later remained) afebrile. PCR levels gradually lowered and WBC and PCT remained normal. Antibiotics were discontinued on day 8 following surgery. 


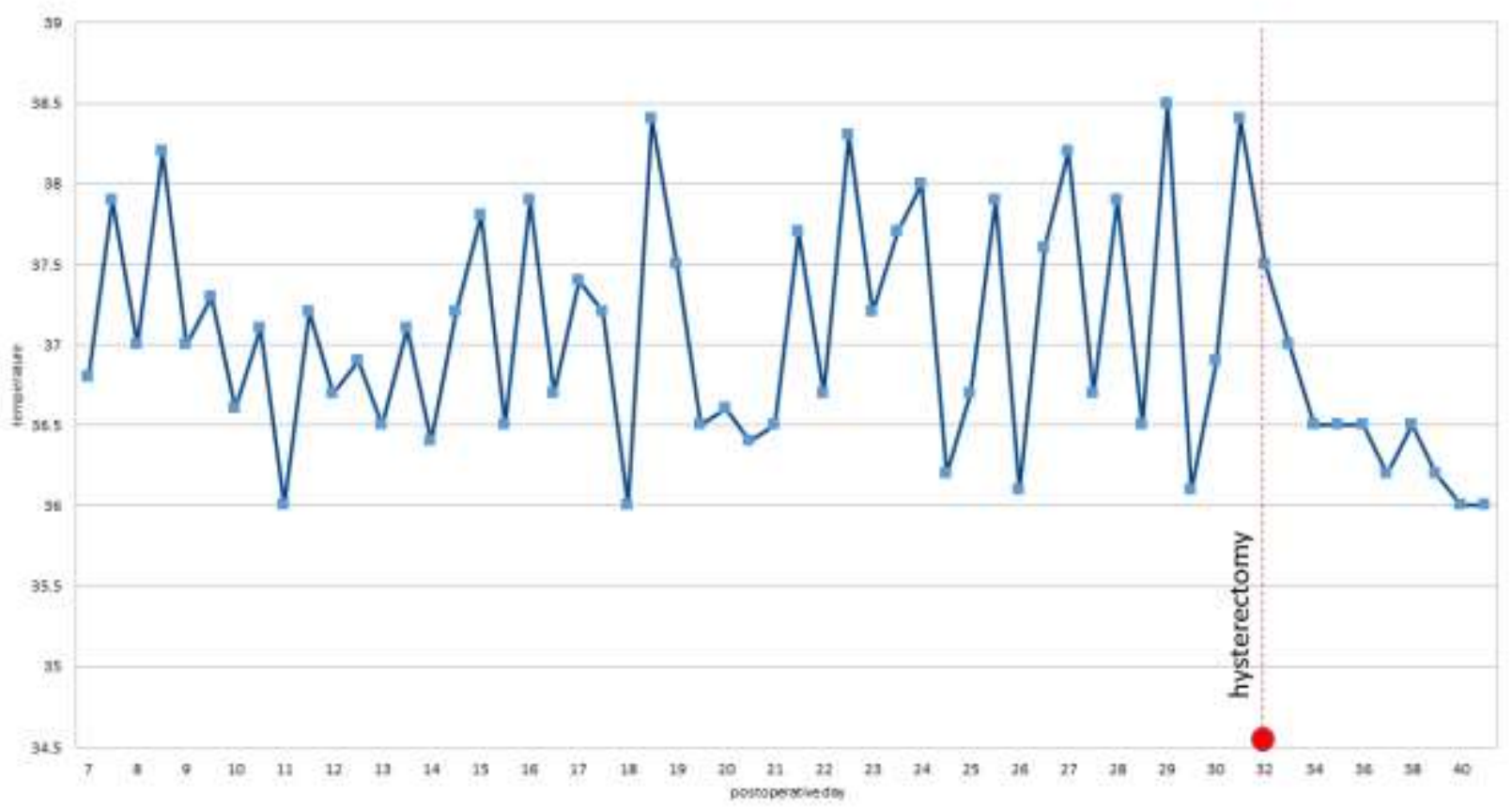

Figure 1: Body temperature throughout the hospital stay.

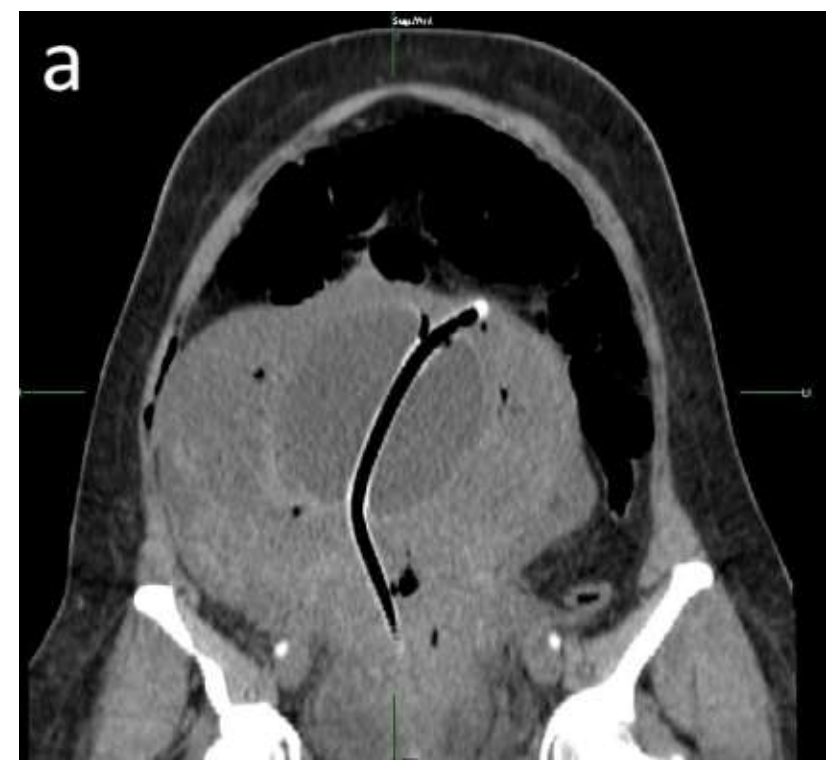

Figure 2a: Coronal multiplanar reconstruction (MPR) of the first CT scan: the uterine cavity is occupied by the Bakri balloon, and some air is visible both near the device and in the myometrium.

\section{DISCUSSION}

Hemorrhagic uterine necrosis or uterine apoplexy is a rare condition first described by Couvelaire. ${ }^{1}$ Couvelaire's historical report referred to a condition of massive uterine infarction following placental abruption. Ischemic necrosis of the uterus has also been described, as a consequence of embolization or surgical ligation of vessels. ${ }^{2,10}$ In this case, we report of a case of massive hemorrhagic infarction of the uterus, followed by uterine necrosis, in a patient with full uterine atony where the uterine arteries were ligated and a uterine compression was performed.

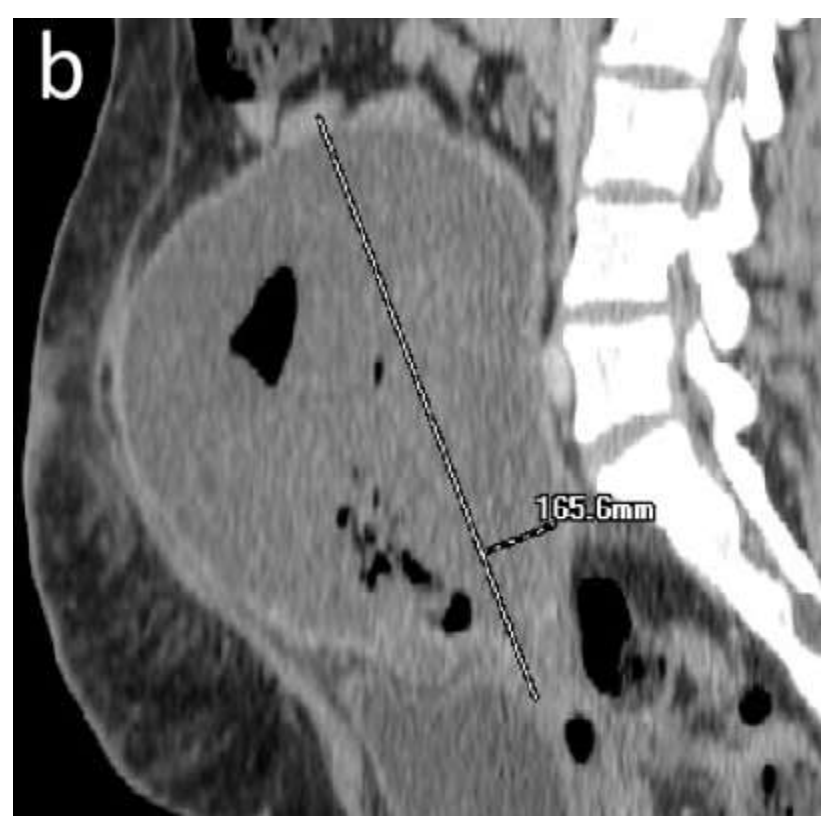

Figure 2b: Sagittal multiplanar reconstruction (MPR) of the second CT scan, showing the abnormal increased dimension of the uterus at 21st day after Csection. Disomogenous hypodense material associated with the presence of air content is also well visible. 


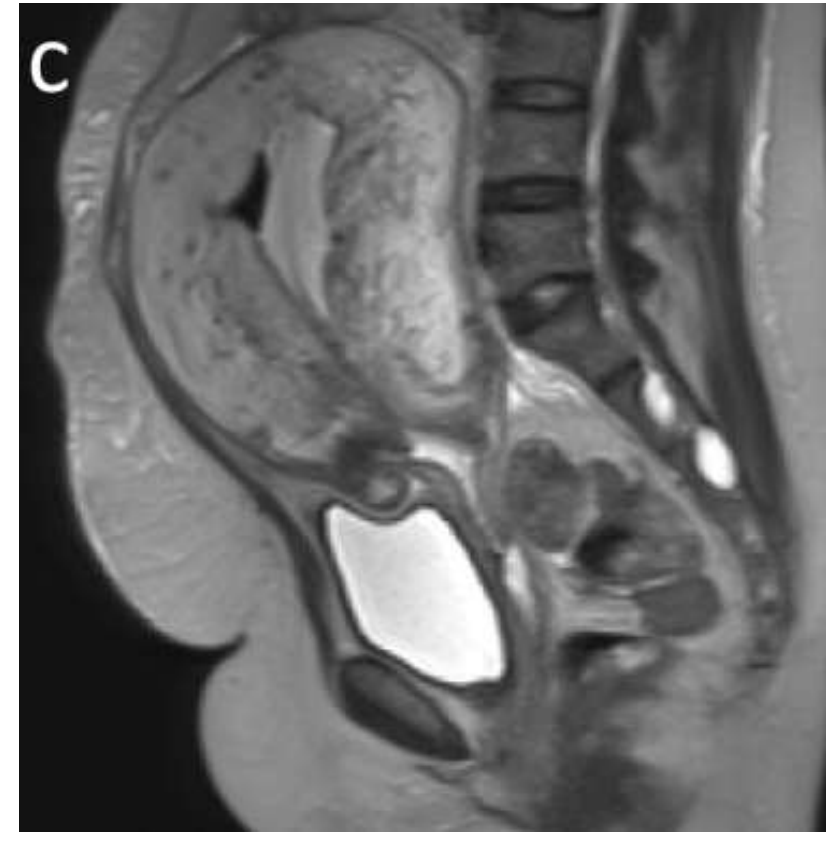

Figure 2c: MRI, sagittal T2w HASTE image: The uterine wall was brightly and diffusely hyperintense due to the complete substitution of the muscular myometrial tissue with hemorrhagic material. No signal intensity typical of the myometrial tissue is appreciable. Some gas is still present inside the endometrial cavity.

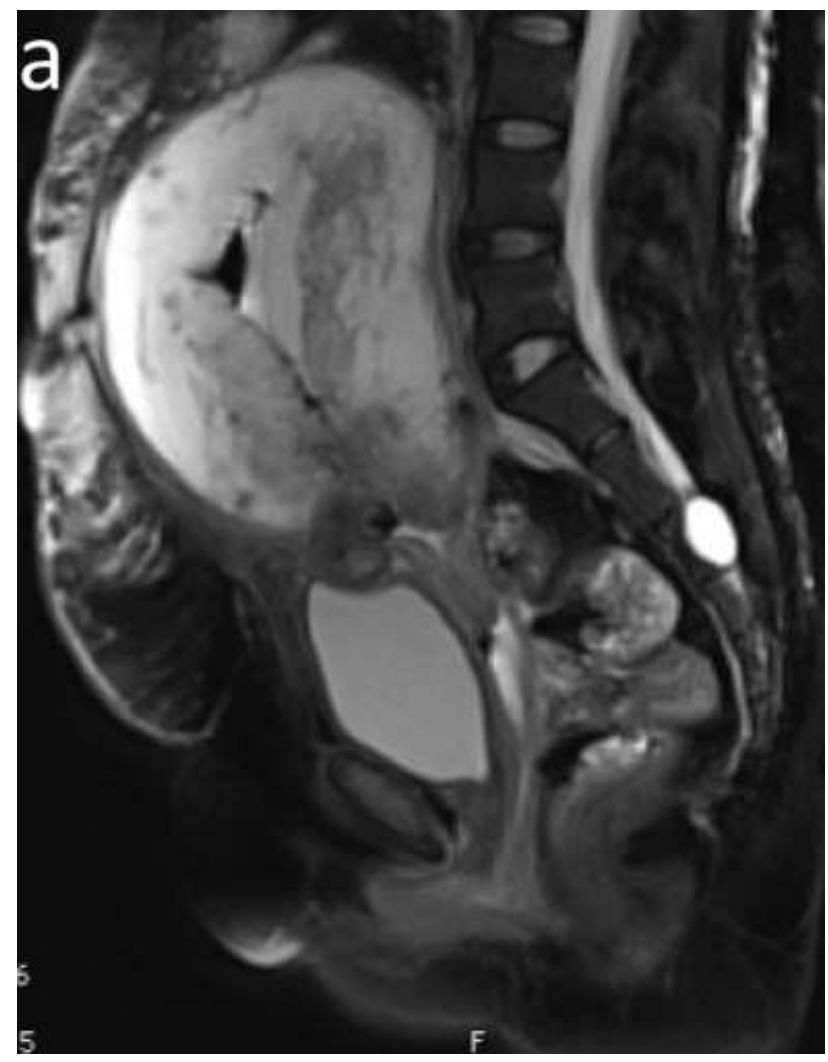

Figure 3a: Sagittal T2 w fat sat sequence highlights a water-enriched myometrium with complete replacement of its musculature.

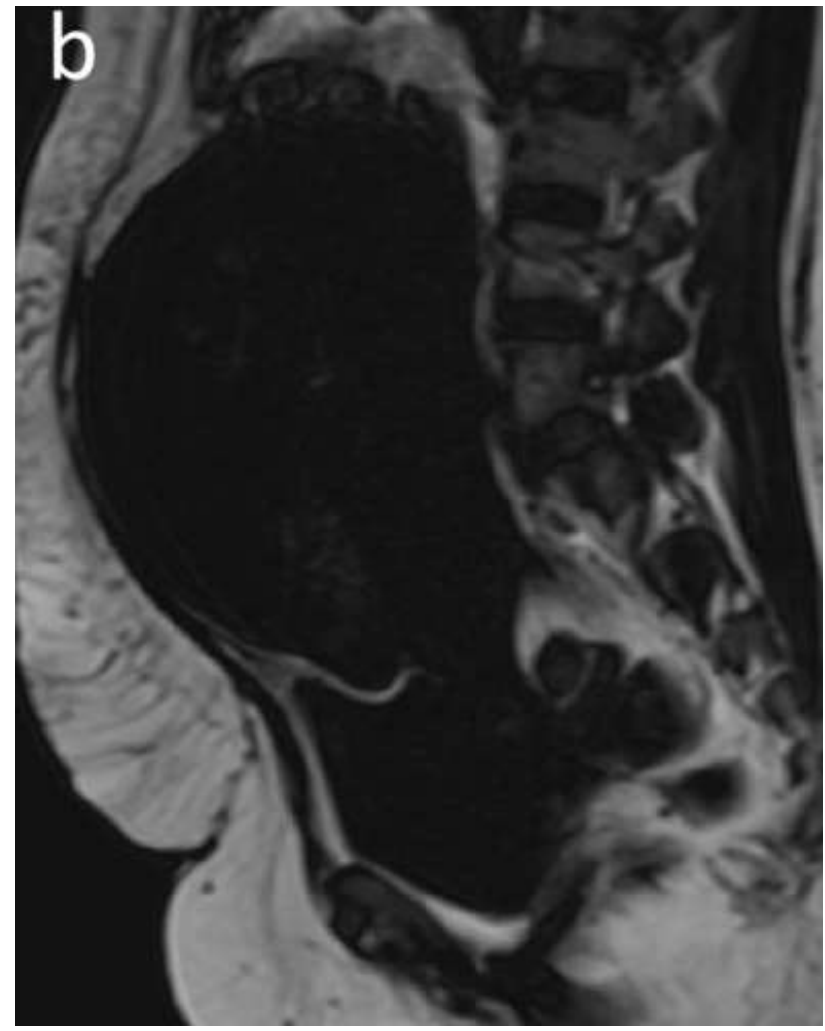

Figure 3b: MRI, sagittal Dixon fat image: the uterine wall is completely hypodense demonstrating the prevalence of water content.

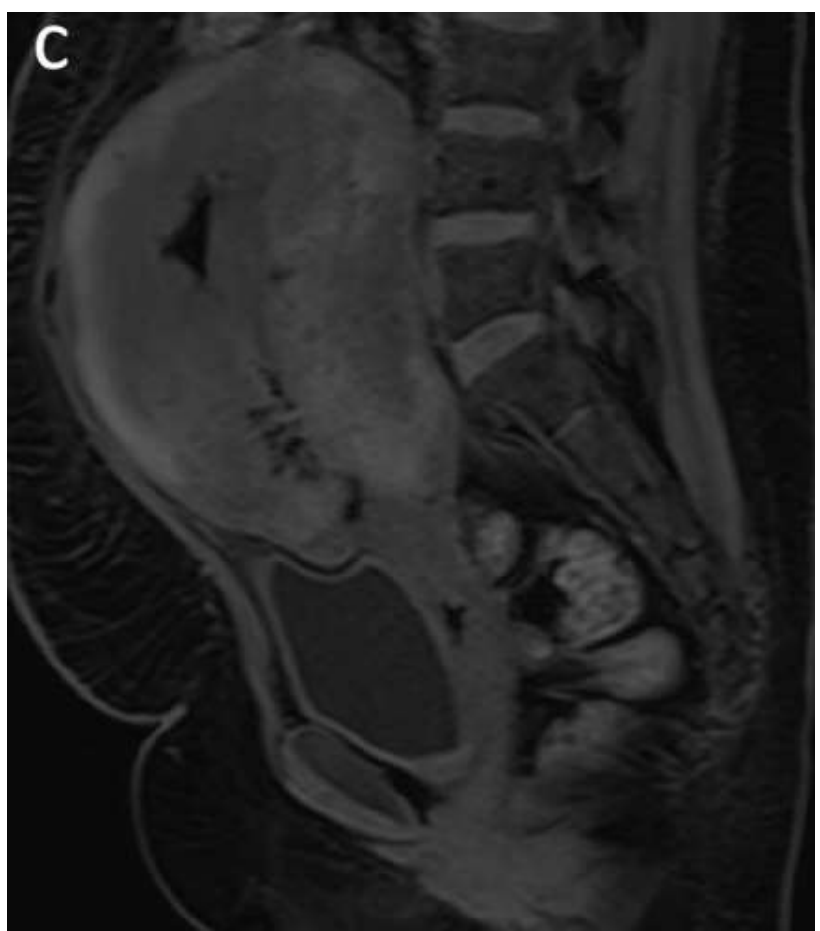

Figure 3c: MRI, sagittal Dixon Water image: the uterine wall shows slightly high signal intensity, demonstrating hemorrhagic content. Some air is visible as signal void spots within myometrium and endometrial cavity. 


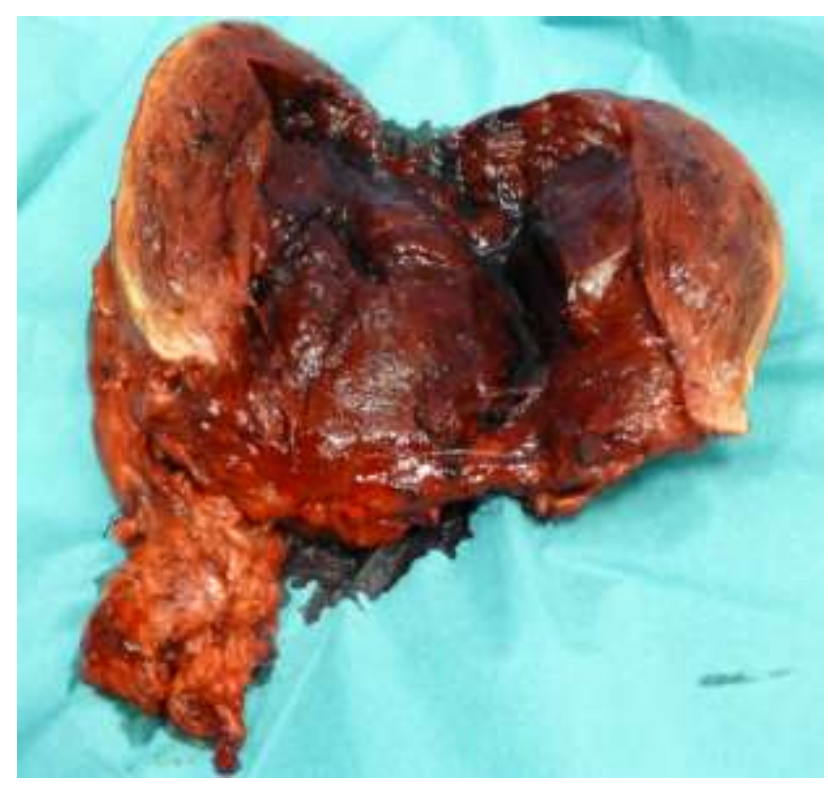

Figure 4: Surgical specimen showing hemorrhagic uterine necrosis with infarcted myometrium.

Diagnosis of this rare condition is difficult, and starts from a correct differentiation of the pathological findings from the normal appearance of the post-gravidic uterus. In the early postpartum period, the uterus is enlarged to twice its normal pre-pregnancy dimensions and then gradually returns to its normal size within 6-8 weeks. The postpartum uterine cavity contains a small amount of fluid and blood debris depicted in US as echogenic intracavitary material and in CT scans as a central area of low attenuation; fresh blood clots are seen as hyper attenuating foci within the central area. The entire postpartum uterus can appear hypervascular on color Doppler images. Intrauterine gas may be seen in the early postpartum period and may persist for as long as 3 weeks as a physiologic finding in the absence of other clinical symptoms. ${ }^{11-13}$

In our case the uterine enlargement did not change in three weeks, as seen on ultrasounds and on CT scans, and the gas content was also intraparietal as well as inside the uterine cavity. The CT attenuation of the myometrium was irregular as previously seen in the literature. ${ }^{5}$

Post contrast enhancement is the key to recognize a normal uterus from a necrotic one, as the literature states for uterine necrosis. ${ }^{2,4}$ The normal postgravidic uterus shows a normal enhancement of its wall, while the necrotic tissue has none.

In our case the critical imaging modality to decide for hysterectomy in this patient, in whom parity and age made the choice of treatment difficult, was MRI. This examination was conducted with a $1.5 \mathrm{~T}$ closed-bore scan (Siemens TIM System, Erlangen, Germany). We performed T2w high speed TSE (HASTE) sequences without fat suppression, T1w fast spoiled GRE (VIBE) sequence with fat suppression and a $\mathrm{T} 1 \mathrm{w}$ fast spoiled
GRE (3D VIBE) sequences with Dixon algorithm. On $\mathrm{T} 2 \mathrm{w}$ HASTE images the uterine wall had very high irregular signal intensity, with little hyperintense fluid deposit inside the endometrial cavity and signal void spots representative of gas presence both in the cavity and the muscular tissue. On fat-suppressed T1w VIBE images the uterus presented medium-low irregular signal intensity, with some hyperintense spots coherent with hematic infarction.

Normal postpartum uterus would have been more regular, even though gas and fluid presence is possible. The most abnormal finding was the uterine size, too big for the $23^{\text {rd }}$ day after $\mathrm{C}$-section. DIXON dataset helped our diagnosis by showing signal hyperintensity of the uterine wall in the water images. That was highly indicative for colliquative necrosis completely replacing the muscular tissue, which would otherwise have medium signal intensity in such sequence.

Unfortunately, endovenous contrast medium was not administered during our MR protocol because of the urgency and the woman's conditions. However, every other feature was congruent with a diagnosis of uterine necrosis and fitted well with the clinical picture of intermittent, non-antibiotic responsive fever.

Literature considers uterine necrosis a rare severe condition mandating immediate treatment by antibiotics and hysterectomy. The rarity of uterine necrosis combined with concern for the woman's future caused a delay in diagnosis and treatment, but antibiotic coverage made the delay not critical, for it excluded most dangerous infective complications.

All procedures followed were in accordance with the ethical standards of the responsible committee on human experimentation (institutional and national) and with the Declaration of Helsinki 1975, as revised in 2000. Written informed consent was obtained from the patient for publication of this case report and any accompanying images.

\section{CONCLUSION}

Hemorrhagic uterine necrosis is a life-threatening disease and its diagnosis can be particularly challenging. It can be detected with CT by lack of uterine contrast enhancement and potentially a gas-filled uterine cavity. MRI can confirm necrosis by showing fluid degeneration of the myometrium. Failure to recognize a necrotic uterus on imaging can cause delayed hysterectomy, which is mandatory and potentially life-saving.

Funding: No funding sources Conflict of interest: None declared

Ethical approval: The study was approved by the Institutional Ethics Committee of the University of Pisa 


\section{REFERENCES}

1. Couvelaire A. Deux nouvelles observations d'apoplexie utero-placentaire (hemorrhagies retroplacentaires avec infiltration sanguine de la pavoi musculaire de l'uterus). Ann Gynecol Obstet. 1912;9:486.

2. Melenhorst M, Hehenkamp W, de Heer K, Berger F. CT features in uterine necrosis of unknown cause: a case report. Clinical Imaging. 2014; 38:543-6.

3. Torigian DA, Siegelman ES, Terhune KP, Butts SF, Blasco L, Shlansky-Goldberg RD. MRI of uterine necrosis after uterine artery embolization for treatment of uterine leiomyomata. AJR Am J Roentgenol. 2005;184(2):555-9.

4. Poujade O, Daher A, Ballout El Maoula A, Aflak N, Bougeois B, Vilgrain V, et al. Uterine necrosis following pelvic arterial embolisation for postpartum haemorrhage: Case report. J Gynecol Obstet Biol Reprod. 2012;41(6):584-6.

5. Gabriel H, Pinto CM, Kumar M, Nikolaidis P, Miller FH, Weinrach DM, et al. MRI detection of uterine necrosis after uterine artery embolization for fibroid. AJR Am J Roentgenol. 2004;183(3):733-6.

6. Kitamura Y, Ascher SM, Cooper C, Allison SJ, Jha RC, Flick PA, et al. Imaging manifestations of complications associated with uterine artery embolization. Radiographics. 2005;(Suppl 1):S11932

7. Pirard C, Squifflet J, Gilles A, Donnez J. Uterine necrosis and sepsis after vascular embolization and surgical ligation in a patient with postpartum hemorrhage. Fertil Steril. 2002;78:412-3.
8. Courbiere B, Jauffret C, Provansal M, Agostini A, Bartoli JM, Cravello L, et al. Failure of conservative management in postpartum haemorrhage: uterine necrosis and hysterectomy after angiographic selective embolization with gelfoam. Eur J Obstet Gynecol Reprod Biol. 2008;140:291-3.

9. Tseng JJ, Ho JY, Wen MC, Hwang JI. Uterine necrosis associated with acute suppuratives myometritis after angiographic selective embolization for refractory postpartum haemorrhage. Am J Obstet Gynecol. 2011;204:e4-6.

10. Rivlin ME, Carroll CS, Morrison JC. Conservative surgery for uterine incisional necrosis complicating cesarean delivery. Obstet Gynecol. 2004;103(5 Pt 2):1105-8.

11. Sierra A, Burrel M, Sebastia C, Radosevic A, Barrufet M, Albela S, Buñesch L, Domingo MA, Salvador R, Real I. Utility of multidetector CT in severe postpartum hemorrhage. Radiographics. 2012;32(5):1463-81.

12. Rodgers SK1, Kirby CL, Smith RJ, Horrow MM. Imaging after cesarean delivery: acute and chronic complications. Radiographics. 2012;32(6):1693-712.

13. Plunk M, Lee JH, Kani K, Dighe M. Imaging of postpartum complications: a multimodality review. AJR. 2013;200:W143-54.

Cite this article as: Giusti S, Fanti F, Cecchi E, Capriello P, Malacarne E, Marmorato M, et al. Hemorrhagic uterine necrosis after surgical vessel ligation and B-Lynch suture in persistent postcesarean uterine atony: case report and review of literature. Int J Reprod Contracept Obstet Gynecol 2016;5:4046-51. 